\title{
Only negligible deviations from electroneutrality are expected in dendritic spines
}

\section{Boris Barbour iD}

The central aim of the Opinion article by Holcman and Yuste (The new nanophysiology: regulation of ionic flow in neuronal subcompartments. Nat. Rev. Neurosci. 16, 685-692 (2015) ) ${ }^{1}$ is to suggest that revolutionary ionic and electrical behaviour will be identified and understood if we no longer apply the classical constraint of electroneutrality when modelling electrodiffusion in small neuronal structures such as dendritic spines. However, as I have argued online ${ }^{2}$, the authors misunderstand the origin of the electroneutrality approximation and present inconsistent calculations that are irrelevant in a biological context. The result is a misleading and deeply confusing Perspective.

\section{Saline solutions are conductors}

The authors calculate distributions of charges in a dieletric ball, which is intended to approximate a spine head. The relative permittivity of the dielectric is that of water, but its conductivity is zero. The authors only consider a single species of ion. In so doing, they ignore completely the large and nearly balanced concentrations of positive and negative ions (and small molecules) that are present inside and outside mammalian neurons. These ions transform the physiological solutions into conductors and cause shielding of charges over an extremely short distance - the Debye length, which is less than a nanometre at physiological ionic strength ${ }^{3}$. No direct electrostatic interactions can operate much beyond this length. However, by ignoring the background ions, the authors enable direct repulsion over microns, 1000-fold greater distances. These considerations of a single charged species are therefore without apparent relevance to the biological situation.

\section{Unavoidable electroneutrality}

The approximation of electroneutrality greatly simplifies the mathematics of electrodiffusion but is anyway quite accurate under physiological conditions. Moreover, the energies available under biological conditions $(\sim 100 \mathrm{mV})$ make it impossible for large-scale deviations from electroneutrality to be generated. These points can be illustrated by the following calculation. For a spine-like sphere delimited by a typical membrane (with an apparent specific capacitance of $\left.1 \mu \mathrm{F} \mathrm{cm}^{-2}\right)$ and with a typical spine radius $(0.25 \mu \mathrm{m})$, we can calculate the number of electronic charges that are transferred when it is charged by $100 \mathrm{mV}(\sim 5,000)$ and compare it with the number of charges that are contained in the sphere with $300 \mathrm{mM}$ ions ( 12 million). The ratio of net/total charges is thus $\sim 0.0004$. This shows why, for dendritic spines and structures of similar scale, electroneutrality remains a very accurate approximation and why it is so hard to violate under biological conditions - the energy required is simply unavailable. A consequence of the difficulty of driving deviations from electroneutrality is that the net charges shown in Fig. 3b,c of the Opinion article would be unattainable in real life.

\section{Inconsistent boundary conditions}

In Box 1 of the Opinion article, the authors present a system of equations for calculating electrodiffusion in a model spine head, which contains only a "single positive ion species". However, at the boundary $\partial \Omega_{\mathrm{r}}$, "there is no flux of the electric field", which is confirmed by the boundary condition in Eq. 3,

$$
\left.\frac{\partial \varphi}{\partial n}\right|_{\partial \Omega_{\mathrm{r}}}=0,
$$

where $\varphi$ is the voltage and $n$ is a normal to the boundary $\partial \Omega_{\mathrm{r}}$; if the electric field is zero at the boundary, so is its flux. By Gauss's Law, which relates the flux of the electric field at a closed surface to the net charge enclosed, the zero-flux condition implies electroneutrality within the boundary, which is incompatible with the presence of positive charges. (The authors clarify elsewhere that the neck opening $\partial \Omega_{\mathrm{a}}$ was not implemented: "here for simplicity, there is no absorption condition at the spine neck and no flux through the PSD,

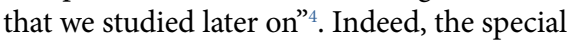
regions for the neck and postsynaptic density do not feature elsewhere in the Perspective.) Thus, in Box 1, the authors appear to imply the existence of electroneutral positive charges! By contrast, the boundary condition in Box 2 does not imply electroneutrality, which makes it inconsistent with that in Box 1. These inconsistencies are not addressed in the Opinion article.

\section{Erroneous capacitance definition}

In Box 2, the authors describe an apparently new and exciting ('non-classical') result regarding the nonlinearity of the membrane capacitance in a nanocompartment. However, there is no membrane in the model. Moreover, the behaviour is 'non-classical' not because of the nanocompartment but because the authors have used a 'non-classical' definition of the capacitance: measured from the centre of the sphere to its boundary, rather than to infinity. It is of no practical application.

\section{Conclusion}

No new insight is gained from these calculations applied to a situation without relevance to biology; they are erroneous and misleading.

Boris Barbour iD

Institut de biologie de l'École normale supérieure (IBENS), École normale supérieure, CNRS, INSERM, PSL University, Paris, France. e-mail: boris.barbour@ens.fr https://doi.org/10.1038/s41583-019-0238-x

1. Holcman, D. \& Yuste, R. The new nanophysiology: regulation of ionic flow in neuronal subcompartments. Nat. Rev. Neurosci. 16, 685-692 (2015).

2. Barbour, B. The electroneutrality liberation front https://referee3.org/2018/08/14/the-electroneutralityliberation-front/ (2018).

3. Hille, B. Ion channels of excitable membranes (Sinauer, 2001).

4. Holcman, D. \& Yuste, R. Answer to B. Barbour fiction-blog about a perspective article:

"The new nanophysiology: regulation of ionic flow in neuronal subcompartments". https://www. ibens.ens.fr/IMG/pdf/response-barbour4_tl_rydh.pdf (2018).

Acknowledgements

The author thanks J. Ranft, V. Hakim and P. Serna for helpful discussion.

Competing interests

The author declares no competing interests. 